Submitted to the Annals of Applied Statistics

arXiv: stat.AP/0812.3671v2

\title{
SUPPLEMENT TO "REGULARIZED MULTIVARIATE REGRESSION FOR IDENTIFYING MASTER PREDICTORS WITH APPLICATION TO INTEGRATIVE GENOMICS STUDY OF BREAST CANCER"
}

\author{
By Jie PenG ${ }^{\dagger}$ Ji Zhu ${ }^{\ddagger}$ AnNaBergamaschi ${ }^{\S}$ WonshikHan $₫$ \\ Dong-YoungNoh ${ }^{\llbracket}$ Jonathan R. Pollack $\|$ Pei Wang**,* \\ ${ }^{\dagger}$ Department of Statistics, University of California, Davis, CA, USA \\ $\ddagger$ Department of Statistics, University of Michigan, Ann Arbor, MI, USA \\ $\S$ Department of Genetics, Institute for Cancer Research, \\ Rikshospitalet-Radiumhospitalet Medical Center, Oslo, Norway \\ " Cancer Research Institute and Department of Surgery, Seoul National \\ University College of Medicine, Seoul, South Korea \\ "Department of Pathology, Stanford University, CA, USA; \\ ** Division of Public Health Science, Fred Hutchinson Cancer Research \\ Center, Seattle, WA, USA.
}

Supplement A: Proof of Theorem 2.1. Define

$$
L(\beta ; Y, X)=\frac{1}{2} \sum_{q=1}^{Q}\left(y_{q}-x \beta_{q}\right)^{2}+\lambda_{1} \sum_{q=1}^{Q}\left|\beta_{q}\right|+\lambda_{2} \sqrt{\sum_{q=1}^{Q} \beta_{q}^{2}} .
$$

It is obvious that, in order to prove Theorem 2.1 in the paper, we only need to show that, the solution of $\min _{\beta} L(\beta ; Y, X)$, is given by (for $q=1, \cdots, Q$ )

$$
\widehat{\beta}_{q}= \begin{cases}0, & \text { if }\left\|\widehat{\beta}^{\text {lasso }}\right\|_{2}=0 \\ \widehat{\beta}_{q}^{\text {lasso }}\left(1-\frac{\lambda_{2}}{\left\|\widehat{\beta}_{\text {lasso }}\right\|_{2} x^{2}}\right)_{+}, & \text {otherwise }\end{cases}
$$

where

$$
\widehat{\beta}_{q}^{\text {lasso }}=\left(1-\frac{\lambda_{1}}{\left|x y_{q}\right|}\right)_{+} \frac{x y_{q}}{x^{2}} .
$$

In the following, for function $L$, view $\left\{\beta_{q^{\prime}}: q^{\prime} \neq q\right\}$ as fixed. With a slight abuse of notation, write $L=L\left(\beta_{q}\right)$. Then when $\beta_{q} \geq 0$, we have

$$
\frac{d L}{d \beta_{q}}=-x y_{q}+\left(x^{2}+\frac{\lambda_{2}}{\|\beta\|_{2}}\right) \beta_{q}+\lambda_{1} \text {. }
$$

\footnotetext{
*Corresponding author, pwang@fhcrc.org.
} 
Thus, $\frac{d L}{d \beta_{q}}>0$ if and only if $\beta_{q}>\tilde{\beta}_{q}^{+}$, where

$$
\tilde{\beta}_{q}^{+}:=\frac{x y_{q}}{x^{2}+\frac{\lambda_{2}}{\|\beta\|_{2}}}\left(1-\frac{\lambda_{1}}{x y_{q}}\right) .
$$

Denote the minima of $\left.L\left(\beta_{q}\right)\right|_{\beta_{q} \geq 0}$ by $\beta_{q, \min }^{+}$. Then, when $\tilde{\beta}_{q}^{+}>0, \beta_{q, \min }^{+}=$ $\tilde{\beta}_{q}^{+}$. On the other hand, when $\tilde{\beta}_{q}^{+} \leq 0, \beta_{q, \min }^{+}=0$. Note that $\tilde{\beta}_{q}^{+}>0$ if and only if $x y_{q}\left(1-\frac{\lambda_{1}}{x y_{q}}\right)>0$. Thus we have

$$
\beta_{q, \min }^{+}= \begin{cases}\tilde{\beta}_{q}^{+}, & \text {if } x y_{q}\left(1-\frac{\lambda_{1}}{x y_{q}}\right)>0 \\ 0, & \text { if } x y_{q}\left(1-\frac{\lambda_{1}}{x y_{q}}\right) \leq 0\end{cases}
$$

Similarly, denote the minima of $\left.L\left(\beta_{q}\right)\right|_{\beta_{q} \leq 0}$ by $\beta_{q, \min }^{-}$, and define

$$
\tilde{\beta}_{q}^{-}:=\frac{x y_{q}}{x^{2}+\frac{\lambda_{2}}{\|\beta\|_{2}}}\left(1+\frac{\lambda_{1}}{x y_{q}}\right)
$$

Then we have

$$
\beta_{q, \min }^{-}= \begin{cases}\tilde{\beta}_{q}^{-}, & \text {if } x y_{q}\left(1+\frac{\lambda_{1}}{x y_{q}}\right)<0 \\ 0, & \text { if } x y_{q}\left(1+\frac{\lambda_{1}}{x y_{q}}\right) \geq 0\end{cases}
$$

Denote the minima of $L\left(\beta_{q}\right)$ as $\widehat{\beta}_{q}$ (with a slight abuse of notation). From the above, it is obvious that, if $x y_{q}>0$, then $\widehat{\beta}_{q} \geq 0$. Thus $\widehat{\beta}_{q}=\max \left(\tilde{\beta}_{q}^{+}, 0\right)=$ $\frac{x y_{q}}{x^{2}+\frac{\left.\lambda_{2}\right|_{2}}{\|\beta\|_{2}}}\left(1-\frac{\lambda_{1}}{x y_{q}}\right)_{+}=\frac{x y_{q}}{x^{2}+\frac{\lambda_{2}}{\|\beta\|_{2}}}\left(1-\frac{\lambda_{1}}{\mid x y_{q}}\right)_{+}$. Similarly, if $x y_{q} \leq 0$, then $\widehat{\beta}_{q} \leq 0$, and it has the same expression as above. Denote the minima of $\left.L(\beta)\right|_{\|\beta\|_{2}>0}$ (now viewed as a function of $\left.\left(\beta_{1}, \cdots, \beta_{Q}\right)\right)$ as $\widehat{\beta}_{\text {min }}=\left(\widehat{\beta}_{1, \text { min }}, \cdots, \widehat{\beta}_{Q, \text { min }}\right)$. We have shown above that, if such a minima exists, it satisfies (for $q=1, \cdots, Q$ )

$$
\widehat{\beta}_{q, \min }=\frac{x y_{q}}{x^{2}+\frac{\lambda_{2}}{\left\|\widehat{\beta}_{\min }\right\|_{2}}}\left(1-\frac{\lambda_{1}}{\left|x y_{q}\right|}\right)_{+}=\widehat{\beta}_{q}^{\text {lasso }} \frac{x^{2}}{x^{2}+\frac{\lambda_{2}}{\left\|\widehat{\beta}_{\min }\right\|_{2}}}
$$

where $\widehat{\beta}_{q}^{\text {lasso }}$ is defined by equation (S-1). Thus

$$
\left\|\widehat{\beta}_{\min }\right\|_{2}=\left\|\widehat{\beta}^{\text {lasso }}\right\|_{2} \frac{x^{2}}{x^{2}+\frac{\lambda_{2}}{\left\|\widehat{\beta}_{\min }\right\|_{2}}} .
$$

By solving the above equation, we obtain

$$
\left\|\widehat{\beta}_{\min }\right\|_{2}=\left\|\widehat{\beta}^{\text {lasso }}\right\|_{2}-\frac{\lambda_{2}}{x^{2}}
$$


By plugging the expression on the right hand side into (S-2), we achieve

$$
\widehat{\beta}_{q, \min }=\widehat{\beta}_{q}^{\text {lasso }}\left(1-\frac{\lambda_{2}}{\left\|\widehat{\beta}^{\text {lasso }}\right\|_{2} x^{2}}\right) .
$$

Denote the minima of $L(\beta)$ by $\widehat{\beta}=\left(\widehat{\beta}_{1}, \cdots, \widehat{\beta}_{Q}\right)$. From the above, we also know that if $\left\|\widehat{\beta}^{\text {lasso }}\right\|_{2}-\frac{\lambda_{2}}{x^{2}}>0, L(\beta)$ achieves its minimum on $\|\beta\|_{2}>0$, which is $\widehat{\beta}=\widehat{\beta}_{\text {min }}$. Otherwise, $L(\beta)$ achieves its minimum at zero. Since $\left\|\widehat{\beta}^{\text {lasso }}\right\|_{2}-\frac{\lambda_{2}}{x^{2}}>0$ if and only if $1-\frac{\lambda_{2}}{\left\|\widehat{\beta}^{\text {lasso }}\right\|_{2} x^{2}}>0$, we have proved the theorem.

Supplement B: BIC criterion for tuning. In this section, we describe the BIC criterion for selecting $\left(\lambda_{1}, \lambda_{2}\right)$. We also derive an unbiased estimator of the degrees of freedom of the remMap estimator under orthogonal design.

In the multivariate regression model

$$
y_{q}=\sum_{p=1}^{P} x_{p} \beta_{p q}+\epsilon_{q}, \quad q=1, \cdots, Q,
$$

by assuming $\epsilon_{q} \sim \operatorname{Normal}\left(0, \sigma_{q, \epsilon}^{2}\right)$, the BIC criterion for the $q^{\text {th }}$ regression can be defined as

$$
\mathrm{BIC}_{q}\left(\widehat{\beta}_{1 q}, \cdots, \widehat{\beta}_{P q} ; \mathrm{df}_{q}\right)=N \times \log \left(\mathrm{RSS}_{q}\right)+\log N \times \mathrm{df}_{q}
$$

where $\operatorname{RSS}_{q}:=\sum_{n=1}^{N}\left(y_{q}^{n}-\widehat{y}_{q}^{n}\right)^{2}$ with $\widehat{y}_{q}^{n}=\sum_{p=1}^{P} x_{p}^{n} \widehat{\beta}_{p q}$; and $\mathrm{df}_{q}$ is the degrees of freedom which is defined as

$$
\mathrm{df}_{q}=\operatorname{df}_{q}\left(\widehat{\beta}_{1 q}, \cdots, \widehat{\beta}_{P q}\right):=\sum_{n=1}^{N} \operatorname{Cov}\left(\widehat{y}_{q}^{n}, y_{q}^{n}\right) / \sigma_{q, \epsilon}^{2},
$$

where $\sigma_{q, \epsilon}^{2}$ is the variance of $\epsilon_{q}$.

For a given pair of $\left(\lambda_{1}, \lambda_{2}\right)$, We then define the (overall) BIC criterion at $\left(\lambda_{1}, \lambda_{2}\right)$ :

$$
\begin{aligned}
\operatorname{BIC}\left(\lambda_{1}, \lambda_{2}\right)= & N \times \sum_{q=1}^{Q} \log \left(\operatorname{RSS}_{q}\left(\lambda_{1}, \lambda_{2}\right)\right) \\
& +\log N \times \sum_{q=1}^{Q} \mathrm{df}_{q}\left(\lambda_{1}, \lambda_{2}\right) .
\end{aligned}
$$

Efron et al. (2004) derive an explicit formula for the degrees of freedom of lars under orthogonal design. Similar strategy are also used by Yuan and Lin (2006) among others. In the following theorem, we follow the same idea and derive an unbiased estimator of $d f_{q}$ for remMap when the columns of $\mathbf{X}$ are orthogonal to each other. 
Theorem 0.1. Suppose $X_{p}^{T} X_{p^{\prime}}=0$ for all $1 \leq p \neq p^{\prime} \leq P$. Then for given $\left(\lambda_{1}, \lambda_{2}\right)$,

$$
\begin{array}{ll}
\widehat{d f}_{q}\left(\lambda_{1}, \lambda_{2}\right) & :=\sum_{p=1}^{P} c_{p q} \times \mathbb{I}\left(\left\|\widehat{B}_{p}^{\text {lasso }}\right\|_{2, C}>\frac{\lambda_{2}}{\left\|X_{p}\right\|_{2}^{2}}\right) \times \mathbb{I}\left(\left|\widehat{\beta}_{p q}^{\text {ols }}\right|>\frac{\lambda_{1}}{\left\|X_{p}\right\|_{2}^{2}}\right) \\
(\mathrm{S}-6) & \times\left(1-\frac{\lambda_{2}}{\left\|X_{p}\right\|_{2}^{2}} \frac{\left\|\widehat{B}_{p}^{\text {lasso }}\right\|_{2, C}^{2}-\left(\widehat{\beta}_{p q}^{\text {lasso }}\right)^{2}}{\left\|\widehat{B}_{p}^{\text {lasso }}\right\|_{2, C}^{3}}\right)+\sum_{p=1}^{P}\left(1-c_{p, q}\right)
\end{array}
$$

is an unbiased estimator of the degrees of freedom $\operatorname{df}_{q}\left(\lambda_{1}, \lambda_{2}\right)$ (defined in equation (S-4)) of the remMap estimator $\widehat{\mathbf{B}}=\widehat{\mathbf{B}}\left(\lambda_{1}, \lambda_{2}\right)=\left(\widehat{\beta}_{p q}\left(\lambda_{1}, \lambda_{2}\right)\right)$.

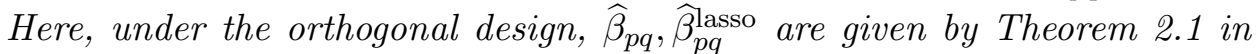
the paper with $\tilde{Y}_{q}=Y_{q}(q=1, \cdots, Q)$, and $\widehat{\beta}_{p q}^{\text {ols }}:=\frac{X_{p}^{T} Y_{q}}{\left\|X_{p}\right\|_{2}^{2}}$.

Before proving Theorem 0.1, we first explain definition (S-4) - the degrees of freedom. Consider the $q^{\text {th }}$ regression $y_{q}=\sum_{p=1}^{P} x_{p} \beta_{p q}+\epsilon_{q}$. Suppose that $\left\{\widehat{y}_{q}^{n}\right\}_{n=1}^{N}$ are the fitted values by a certain fitting procedure based on the current observations $\left\{y_{q}^{n}: n=1, \cdots, N ; q=1, \cdots, Q\right\}$. Let $\mu_{q}^{n}:=\sum_{p=1}^{P} x_{p}^{n} \beta_{p q}$. Then for a fixed design matrix $X=\left(x_{p}^{n}\right)$, the expected re-scaled prediction error of $\left\{\widehat{y}_{q}^{n}\right\}_{n=1}^{N}$ in predicting a future set of new observations $\left\{\widetilde{y}_{q}^{n}\right\}_{n=1}^{N}$ from the $q^{\text {th }}$ regression is:

$$
\mathrm{PE}_{q}=\sum_{n=1}^{N} E\left(\left(\widehat{y}_{q}^{n}-\widehat{y}_{q}^{n}\right)^{2}\right) / \sigma_{q, \epsilon}^{2}=\sum_{n=1}^{N} E\left(\left(\widehat{y}_{q}^{n}-\mu_{q}^{n}\right)^{2}\right) / \sigma_{q, \epsilon}^{2}+N .
$$

Note that

$$
\left(\widehat{y}_{q}^{n}-y_{q}^{n}\right)^{2}=\left(\widehat{y}_{q}^{n}-\mu_{q}^{n}\right)^{2}+\left(y_{q}^{n}-\mu_{q}^{n}\right)^{2}-2\left(\widehat{y}_{q}^{n}-\mu_{q}^{n}\right)\left(y_{q}^{n}-\mu_{q}^{n}\right) .
$$

Therefore,

$$
\mathrm{PE}_{q}=\sum_{n=1}^{N} E\left(\left(\widehat{y}_{q}^{n}-y_{q}^{n}\right)^{2}\right) / \sigma_{q, \epsilon}^{2}+2 \sum_{n=1}^{N} \operatorname{Cov}\left(\widehat{y}_{q}^{n}, y_{q}^{n}\right) / \sigma_{q, \epsilon}^{2} .
$$

Denote $R S S_{q}=\sum_{n=1}^{N}\left(\widehat{y}_{q}^{n}-y_{q}^{n}\right)^{2}$. Then an un-biased estimator of $\mathrm{PE}_{q}$ is

$$
R S S_{q} / \sigma_{q, \epsilon}^{2}+2 \sum_{n=1}^{N} \operatorname{Cov}\left(\widehat{y}_{q}^{n}, y_{q}^{n}\right) / \sigma_{q, \epsilon}^{2} .
$$

Therefore, a natural definition of the degrees of freedom for the procedure resulting the fitted values $\left\{\widehat{y}_{q}^{n}\right\}_{n=1}^{N}$ is as given in equation (S-4). Note that, this is the definition used in Mallow's $C_{p}$ criterion. 
Proof of Theorem 0.1: By applying Stein's identity to the Normal distribution, we have: if $Z \sim N\left(\mu, \sigma^{2}\right)$, and a function $g$ such that $E\left(\left|g^{\prime}(Z)\right|\right)<\infty$, then

$$
\operatorname{Cov}(g(Z), Z) / \sigma^{2}=E\left(g^{\prime}(Z)\right)
$$

Therefore, under the normality assumption on the residuals $\left\{\epsilon_{q}\right\}_{q=1}^{Q}$ in the multivariate regression model, definition ( $\mathrm{S}-4)$ becomes

$$
d f_{q}=\sum_{n=1}^{N} E\left(\frac{\partial \widehat{y}_{q}^{n}}{\partial y_{q}^{n}}\right), \quad q=1, \cdots, Q
$$

Thus an obvious unbiased estimator of $d f_{q}$ is $\widehat{d f}_{q}=\sum_{n=1}^{N} \frac{\partial \widehat{y}_{q}^{n}}{\partial y_{q}^{n}}$. In the following, we derive $\widehat{d f}_{q}$ for the proposed remMap estimator under the orthogonal design. Let $\widehat{\beta}_{q}=\left(\widehat{\beta}_{1 q}, \cdots, \widehat{\beta}_{P q}\right)$ be a one by $P$ row vector; let $\mathbf{X}=\left(x_{p}^{n}\right)$ be the $N$ by $P$ design matrix which is orthonormal; let $Y_{q}=\left(y_{1}^{1}, \cdots, y_{q}^{N}\right)^{T}$ and $\widehat{Y}_{q}=\left(\widehat{y}_{q}^{1}, \cdots, \widehat{y}_{q}^{N}\right)^{T}=\mathbf{X} \widehat{\beta}_{q}$ be $N$ by one column vectors. Then

$$
\widehat{d f}_{q}=\operatorname{tr}\left(\frac{\partial \widehat{Y}_{q}}{\partial Y_{q}}\right)=\operatorname{tr}\left(\mathbf{X} \frac{\partial \widehat{\beta}_{q}}{\partial Y_{q}}\right)=\operatorname{tr}\left(\mathbf{X} \frac{\partial \widehat{\beta}_{q}}{\partial \widehat{\beta}_{q, \mathrm{ols}}} \frac{\partial \widehat{\beta}_{q, \mathrm{ols}}}{\partial Y_{q}}\right)
$$

where $\widehat{\beta}_{q, \text { ols }}=\left(\widehat{\beta}_{1 q}^{\text {ols }}, \cdots, \widehat{\beta}_{P q}^{\text {ols }}\right)^{T}$ and the last equality is due to the chain rule. Since under the orthogonal design, $\widehat{\beta}_{p q}^{\text {ols }}=X_{p}^{T} Y_{q} /\left\|X_{p}\right\|_{2}^{2}$, where $X_{p}=$ $\left(x_{p}^{1}, \cdots, x_{p}^{N}\right)^{T}$, thus $\frac{\partial \widehat{\beta}_{q, \text { ols }}}{\partial Y_{q}}=\mathbf{D} \mathbf{X}^{T}$, where $\mathbf{D}$ is a $P$ by $P$ diagonal matrix with the $p^{t h}$ diagonal entry being $1 /\left\|X_{p}\right\|_{2}^{2}$. Therefore

$\widehat{d f}_{q}=\operatorname{tr}\left(\mathbf{X} \frac{\partial \widehat{\beta}_{q}}{\partial \widehat{\beta}_{q, \mathrm{ols}}} \mathbf{D} \mathbf{X}^{T}\right)=\operatorname{tr}\left(\mathbf{D X}^{T} \mathbf{X} \frac{\partial \widehat{\beta}_{q}}{\partial \widehat{\beta}_{q, \mathrm{ols}}}\right)=\operatorname{tr}\left(\frac{\partial \widehat{\beta}_{q}}{\partial \widehat{\beta}_{q, \mathrm{ols}}}\right)=\sum_{p=1}^{P} \frac{\partial \widehat{\beta}_{p q}}{\partial \widehat{\beta}_{p q}^{\mathrm{ols}}}$,

where the second to last equality is by $\mathbf{X}^{T} \mathbf{X}=\mathbf{D}^{-1}$ which is due to the orthogonality of $\mathbf{X}$. By the chain rule

$$
\frac{\partial \widehat{\beta}_{p q}}{\partial \widehat{\beta}_{p q}^{\mathrm{ols}}}=\frac{\partial \widehat{\beta}_{p q}}{\partial \widehat{\beta}_{p q}^{\text {lasso }}} \frac{\partial \widehat{\beta}_{p q}^{\text {lasso }}}{\partial \widehat{\beta}_{p q}^{\mathrm{ols}}} .
$$

By Theorem 2.1 in the paper, under the orthogonal design,

$$
\frac{\partial \widehat{\beta}_{p q}}{\partial \widehat{\beta}_{p q}^{\text {lasso }}}=\mathbb{I}\left(\left\|\widehat{B}_{p}^{\text {lasso }}\right\|_{2, C}>\frac{\lambda_{2}}{\left\|X_{p}\right\|_{2}^{2}}\right) \times\left[1-\frac{\lambda_{2}}{\left\|X_{p}\right\|_{2}^{2}} \frac{\left\|\widehat{B}_{p}^{\text {lasso }}\right\|_{2, C}^{2}-\left(\widehat{\beta}_{p q}^{\text {lasso }}\right)^{2}}{\left\|\widehat{B}_{p}^{\text {lasso }}\right\|_{2, C}^{3}}\right],
$$


and

$$
\frac{\partial \widehat{\beta}_{p q}^{\text {lasso }}}{\partial \widehat{\beta}_{p q}^{\text {ols }}}= \begin{cases}1, & \text { if } c_{p, q}=0 \\ \mathbb{I}\left(\left|\widehat{\beta}_{p q}^{\text {ols }}\right|>\frac{\lambda_{1}}{\left\|X_{p}\right\|_{2}^{2}}\right), & \text { if } c_{p, q}=1\end{cases}
$$

Note that when $c_{p, q}=0, \widehat{\beta}_{p q}=\widehat{\beta}_{p q}^{\text {ols }}$, thus $\frac{\partial \widehat{\beta}_{p q}}{\partial \widehat{\beta}_{p q}^{\text {ols }}}=1$. It is then easy to show that $\widehat{d f}_{q}$ is as given in equation (S-6).

Note that, when the $\ell_{2}$ penalty parameter $\lambda_{2}$ is 0 , the model becomes $q$ separate lasso regressions with the same penalty parameter $\lambda_{1}$ and the degrees of freedom estimation in equation (S-6) is simply the total number of non-zero coefficients in the model (under orthogonal design). When $\lambda_{2}$ is nonzero, the degrees of freedom of remMap estimator should be smaller than the number of non-zero coefficients due to the additional shrinkage induced by the $\ell_{2}$ norm part of the MAP penalty (equation (2.2) in the paper). This is reflected by equation (S-6).

Supplement C: Additional Simulation. We conduct a simulation study to investigate the scenario where some columns of the coefficient matrix B are dependent. Specifically, we set $\beta_{p, q_{1}}=\beta_{p, q_{2}}$ if $\beta_{p, q_{1}} \neq 0, \beta_{p, q_{2}} \neq 0$, i.e., the effect of predictor $X_{p}$ on related responses are the same. The results are reported in the table below. As we can see, the overall picture of the performances of different methods remains similar as other simulations.

TABLE S-1

New simulation: dependent regression coefficients

\begin{tabular}{c|ccc|cc}
\hline \hline Method & FP & FN & TF & FPP & FNP \\
\hline remMap.bic & 22.64 & 17.80 & 40.44 & 0.00 & 0.72 \\
remMap.cv & 14.08 & 16.00 & 30.08 & 0.24 & 0.48 \\
remMap.cv.vote & 5.16 & 19.56 & 24.72 & 0.04 & 0.52 \\
\hline joint.bic & 2.80 & 42.84 & 45.64 & 2.76 & 0.40 \\
joint.cv & 17.60 & 33.24 & 50.84 & 17.08 & 0.12 \\
joint.cv.vote & 3.00 & 43.60 & 46.6 & 2.96 & 0.36 \\
\hline sep.bic & 76.36 & 27.00 & 103.36 & 70.96 & 0.00 \\
sep.cv & 239.80 & 22.48 & 262.28 & 197.12 & 0.00 \\
sep.cv.vote & 169.36 & 23.08 & 192.44 & 146.96 & 0.00 \\
\hline
\end{tabular}

FP: false positive; FN: false negative; TF: total false;

FPP: false positive trans-predictor;

FNP: false negative trans-predictor.

\section{Supplement D: Data Preprocessing.}


D.1 Preprocessing for array CGH data. Each array output ( $\log _{2}$ ratios) is first standardized to have median $=0$ and smoothed by cghFLasso (Tibshirani and Wang 2008) for defining gained/lost regions on the genome. The noise level of each array is then calculated based on the measurements from the estimated normal regions (i.e., regions with estimated copy numbers equal to 2). After that, each smoothed array is normalized according to its own noise level.

We define copy number alteration intervals (CNAIs) by using the FixedOrder Clustering (FOC) method (Wang 2004). FOC first builds a hierarchical clustering tree along the genome based on all arrays, and then cuts the tree at an appropriate height such that genes with similar copy numbers fall into the same CNAI. FOC is a generalization of the CLAC (CLuster Along Chromosome) method proposed by Wang et al. (2005). It differs in two ways from the standard agglomerative clustering. First, the order of the leaves in the tree is fixed, which represents the genome order of the genes/clones in the array. So, only adjacent clusters are joined together when the tree is generated by a bottom-up approach. Second, the similarity between two clusters no longer refers to the spatial distance but to the similarity of the array measurements ( $\log _{2}$ ratio) between the two clusters. By using FOC, the whole genome is divided into 384 non-overlapping CNAIs based on all 172 CGH arrays. This is illustrated in Figure S-1. In addition, the heatmap of the (sample) correlations of the CNAIs is given in Figure S-2.

D.2 Selection of breast cancer related genes. We combine seven published breast cancer gene lists: the intrinsic gene set (Sorlie et al. 2003), the Amsterdam 70 gene (van de Vijver et al. 2002), the wound response gene set (Chang et al. 2004), the 76 genes for the metastasis signature (Wang et al. 2005), the gene list for calculating the recurrence score (Paik et al. 2004), the gene list of the Genomic Grade Index (GGI) (Sotiriou et al. 2006), and the PTEN gene set (Saal et al. 2007). Among this union set of breast cancer related genes, 967 overlap with the genes in the current expression data set. We further filter away genes with missing measurements in more than $20 \%$ of the samples, and 654 genes are left. Among these 654 selected genes, 449 are from the intrinsic gene set (Sorlie et al. 2003), which are used to derive breast cancer subtype labels in Appendix D.4.

D.3 Interactions among RNA expressions. We apply the space (Sparse PArtial Correlation Estimation) method (Peng et al. 2008) to infer the interactions among RNA levels through identifying non-zero partial correlations. space assumes the overall sparsity of the partial correlation matrix and employs sparse regression techniques for model fitting. As indicated by 
many experiments that genetic-regulatory networks have a power-law type degree distribution with a power parameter between 2 and 3 (Newman 2003), the tuning parameter in space is chosen such that the resulting network has an estimated power parameter around 2 (see Figure S-3(b) for the corresponding degree distribution). The resulting (concentration) network has 664 edges in total, whose topology is illustrated in Figure S-3(a). In this network, there are 7 nodes having at least 10 edges. These hub genes include PLK1, PTTG1, AURKA, ESR1, and GATA3. PLK1 has important functions in maintaining genome stability via its role in mitosis. Its over expression is associated with preinvasive in situ carcinomas of the breast (Rizki et al. 2007). PTTG1 is observed to be a proliferation marker in invasive ductal breast carcinomas (Talvinen et al. 2008). AURKA encodes a cell cycle-regulated kinase and is a potential metastasis promoting gene for breast cancer (Thomassen et al. 2008). ESR1 encodes an estrogen receptor, and is a well known key player in breast cancer. Moreover, it had been reported that GATA3 expression has a strong association with estrogen receptor in breast cancer (Voduc et al. 2008). Detailed annotation of these and other hub genes are listed in Table S-2. We refer to this network as Exp.Net.664, and use it to account for RNA interactions when investigating the regulations between CNAIs and RNA levels.

TABLE S-2

Annotations for hub genes (degrees greater than 10) in the inferred RNA interaction network Exp.Net.664.

\begin{tabular}{c|llll}
\hline \hline CloneID & Gene Name & Symbol & ID & Cytoband \\
\hline 744047 & Polo-like kinase 1 (Drosophila) & PLK1 & 5347 & $16 \mathrm{p} 12.1$ \\
781089 & Pituitary tumor-transforming 1 & PTTG1 & 9232 & $5 \mathrm{q} 35.1$ \\
129865 & Aurora kinase A & AURKA & 6790 & $20 \mathrm{q} 13.2-\mathrm{q} 13.3$ \\
214068 & GATA binding protein 3 & GATA3 & 2625 & $10 \mathrm{p} 15$ \\
950690 & Cyclin A2 & CCNA2 & 890 & $4 \mathrm{q} 25-\mathrm{q} 31$ \\
120881 & RAB31, member RAS oncogene family & RAB31 & 11031 & $18 \mathrm{p} 11.3$ \\
725321 & Estrogen receptor 1 & ESR1 & 2099 & $6 \mathrm{q} 25.1$ \\
\hline \hline
\end{tabular}

D.4 Breast Cancer Subtypes. Population stratification due to distinct subtypes could confound our detection of associations between CNAIs and gene expressions. For example, if the copy number of CNAI A and expression level of gene B are both higher in one subtype than in the other subtypes, we could observe a strong correlation between CNAI A and gene expression B across the whole population, even when the correlation within each subtype is rather weak. To account for this potential confounding factor, we introduce a set of subtype indicator variables, which is used as additional predictors in the remMap model. Specifically, we derive subtype labels based on expression 
patterns by following the work of Sorlie et al. (2003). We first normalize the expression levels of each intrinsic gene (449 in total) across the 172 samples to have mean zero and MAD one. Then we use kmeans clustering to divide the patients into five distinct groups, which correspond to the five subtypes suggested by Sorlie et al. (2003) — Luminal Subtype A, Luminal Subtype B, ERBB2-overexpressing Subtype, Basal Subtype and Normal Breast-like Subtype. Figure S-4 illustrates the expression patterns of these five subtypes across the 172 samples. We then define five dummy variables to represent the subtype information for each tumor sample, and include them as predictors when fitting the remMap model.

D.5 Comments on the results of the remMap analysis.. remMap analysis suggests that the amplification of a trans-hub region on chromosome 17 influences the RNA expression levels of 31 distinct (unlinked) genes. Some of the 31 genes/clones have been reported to have functions directly related to cancer and may serve as potential drug targets. For example, AGTR1 is a receptor whose genetic polymorphisms have been reported to associate with breast cancer risk and is possibly druggable (Koh et al. 2005). CDH3 encodes a cell-cell adhesion glycoprotein and is deemed as a candidate of tumor suppressor gene, as disturbance of intracellular adhesion is important for invasion and metastasis of tumor cells (Kremmidiotis et al. 1998). PEG3 is a mediator between p53 and Bax in DNA damage-induced neuronal death (Johnson et al. 2002) and may function as a tumor suppressor gene (Dowdy et al. 2005). In a word, these 31 genes may play functional roles in the pathogenesis of breast cancer and may serve as additional targets for therapy.

\section{References.}

Chang HY, Sneddon JB, Alizadeh AA, Sood R, West RB, et al., (2004), "Gene expression signature of fibroblast serum response predicts human cancer progression: Similarities between tumors and wounds," PLoS Biol, 2(2).

Dowdy SC, Gostout BS, Shridhar V, Wu X, Smith DI, Podratz KC, Jiang SW., (2005) "Biallelic methylation and silencing of paternally expressed gene 3(PEG3) in gynecologic cancer cell lines," Gynecol Oncol, 99(1), 126-34.

Efron, B., Hastie, T., Johnstone, I., and Tibshirani, R. (2004), "Least Angle Regression," Annals of Statistics, 32, 407-499.

Johnson MD, Wu X, Aithmitti N, Morrison RS., (2002) "Peg3/Pw1 is a mediator between p53 and Bax in DNA damage-induced neuronal 
death," J Biol Chem, 277(25), 23000-7.

Koh WP, Yuan JM, Van Den Berg D, Lee HP, Yu MC., (2005) "Polymorphisms in angiotensin II type 1 receptor and angiotensin I-converting enzyme genes and breast cancer risk among Chinese women in Singapore," Carcinogenesis, 26(2), 459-64.

Kremmidiotis G, Baker E, Crawford J, Eyre HJ, Nahmias J, Callen DF., (1998), "Localization of human cadherin genes to chromosome regions exhibiting cancer-related loss of heterozygosity," Genomics, 49(3), 467-71.

Newman M, (2003), "The Structure and Function of Complex Networks," Society for Industrial and Applied Mathematics, 45(2), 167-256.

Paik S, Shak S, Tang G, Kim C, Baker J, et al., (2004), "A multigene assay to predict recurrence of tamoxifen-treated, node-negative breast cancer," N Engl J Med, 351(27), 2817-2826.

Peng, J., P. Wang, N. Zhou, J. Zhu, (2008), "Partial Correlation Estimation by Joint Sparse Regression Models," JASA, 104 (486).

Rizki A, Mott JD, Bissell MJ, (2007) "Polo-like kinase 1 is involved in invasion through extracellular matrix," Cancer Res, 67(23), 11106-10.

Saal LH, Johansson P, Holm K, Gruvberger-Saal SK, She QB, et al., (2007), "Poor prognosis in carcinoma is associated with a gene expression signature of aberrant PTEN tumor suppressor pathway activity," Proc Natl Acad Sci U S A, 104(18), 7564-7569.

Sorlie, T., Tibshirani, R., Parker, J., Hastie, T., Marron, J. S., Nobel, A., Deng, S., Johnsen, H., Pesich, R., Geisler, S., Demeter,J., Perou,C. M., Lnning, P. E., Brown, P. O., Brresen-Dale, A.-L., and Botstein, D., (2003), "Repeated observation of breast tumor subtypes in independent gene expression data sets," Proc Natl Acad Sci U S A, 100, 84188423.

Sotiriou C, Wirapati P, Loi S, Harris A, Fox S, et al.,(2006), "Gene expression profiling in breast cancer: Understanding the molecular basis of histologic grade to improve prognosis," J Natl Cancer Inst, 98(4), 262272.

Talvinen K, Tuikkala J, Nevalainen O, Rantanen A, Hirsimäki P, Sundström J, Kronqvist P, (2008), "Proliferation marker securin identifies favourable outcome in invasive ductal breast cancer," $\mathrm{Br} J \mathrm{Can}$ cer, 99(2), 335-40.

Thomassen M, Tan Q, Kruse TA, (2008) "Gene expression meta-analysis identifies chromosomal regions and candidate genes involved in breast cancer metastasis," Breast Cancer Res Treat, Feb 22, Epub.

Tibshirani, R. and Wang, P., (2008) "Spatial smoothing and hot spot 
detection for cgh data using the fused lasso," Biostatistics , 9(1), 1829.

van de Vijver MJ, He YD, van’t Veer LJ, Dai H, Hart AA, Voskuil DW, Schreiber GJ, Peterse JL, Roberts C, Marton MJ, Parrish M, Atsma D, Witteveen A, Glas A, Delahaye L, van der Velde T, Bartelink H, Rodenhuis S, Rutgers ET, Friend SH, Bernards R, (2002) "A geneexpression signature as a predictor of survival in breast cancer," $N$ Engl J Med, 347(25), 1999-2009.

Voduc D, Cheang M, Nielsen T, (2008), "GATA-3 expression in breast cancer has a strong association with estrogen receptor but lacks independent prognostic value," Cancer Epidemiol Biomarkers Prev, 17(2), 365-73.

Wang, P., (2004) "Statistical methods for CGH array analysis," Ph.D. Thesis, Stanford University, 80-81.

Wang P, Kim Y, Pollack J, Narasimhan B, Tibshirani R, (2005) "A method for calling gains and losses in array CGH data," Biostatistics, 6(1), 45-58.

Wang Y, Klijn JG, Zhang Y, Sieuwerts AM, Look MP, et al.,(2005), "Gene-expression profiles to predict distant metastasis of lymph-nodenegative primary breast cancer," Lancet, 365(9460), 671-679.

Yuan, M. and Lin, Y., (2006) "Model Selection and Estimation in Regression with Grouped Variables," Journal of the Royal Statistical Society, Series B, 68(1), 49-67. 


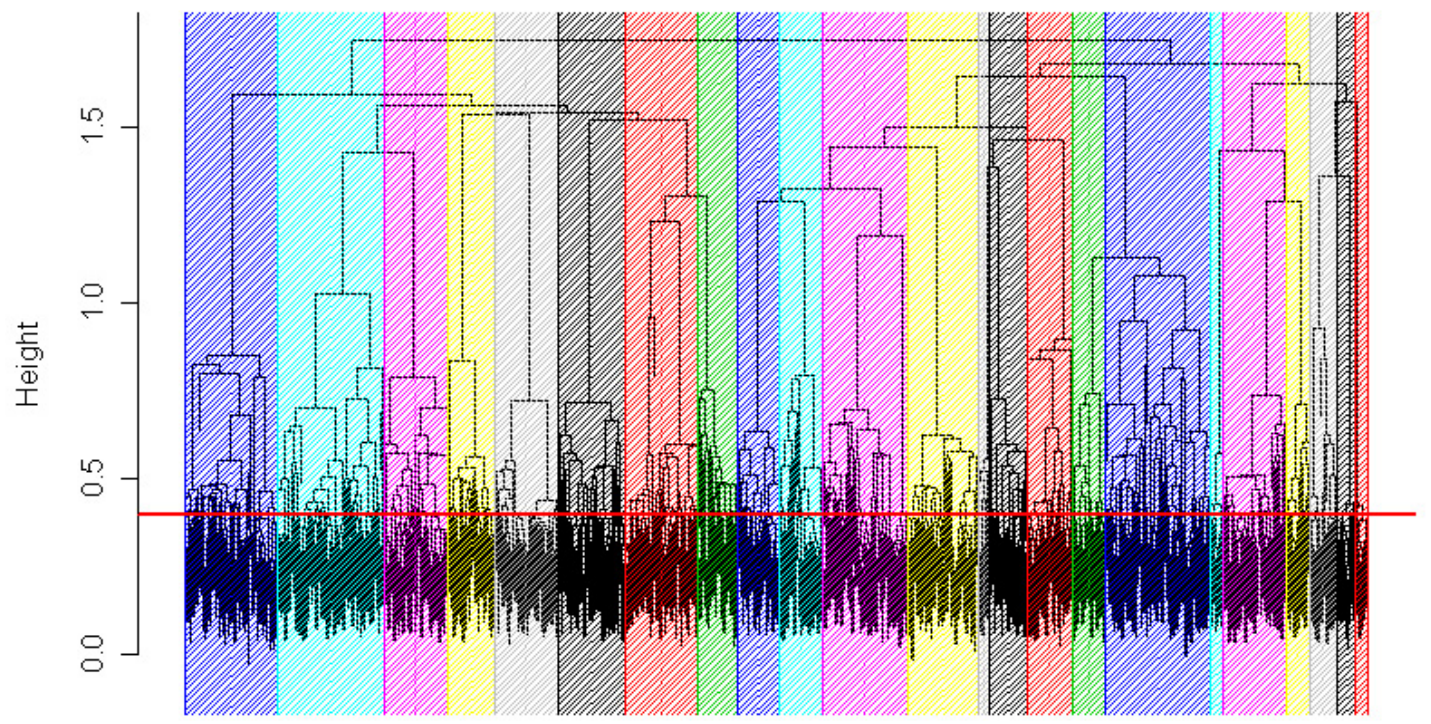

FiG S-1. Hierarchical tree constructed by FOC. Each leaf represents one gene/clone on the array. The order of the leaves represents the order of genes on the genome. The 23 Chromosomes are illustrated with different colors. Cutting the tree at 0.04 (horizonal red line) separates the genome into 384 intervals. This cutoff point is chosen such that no interval contains genes from different chromosomes. 

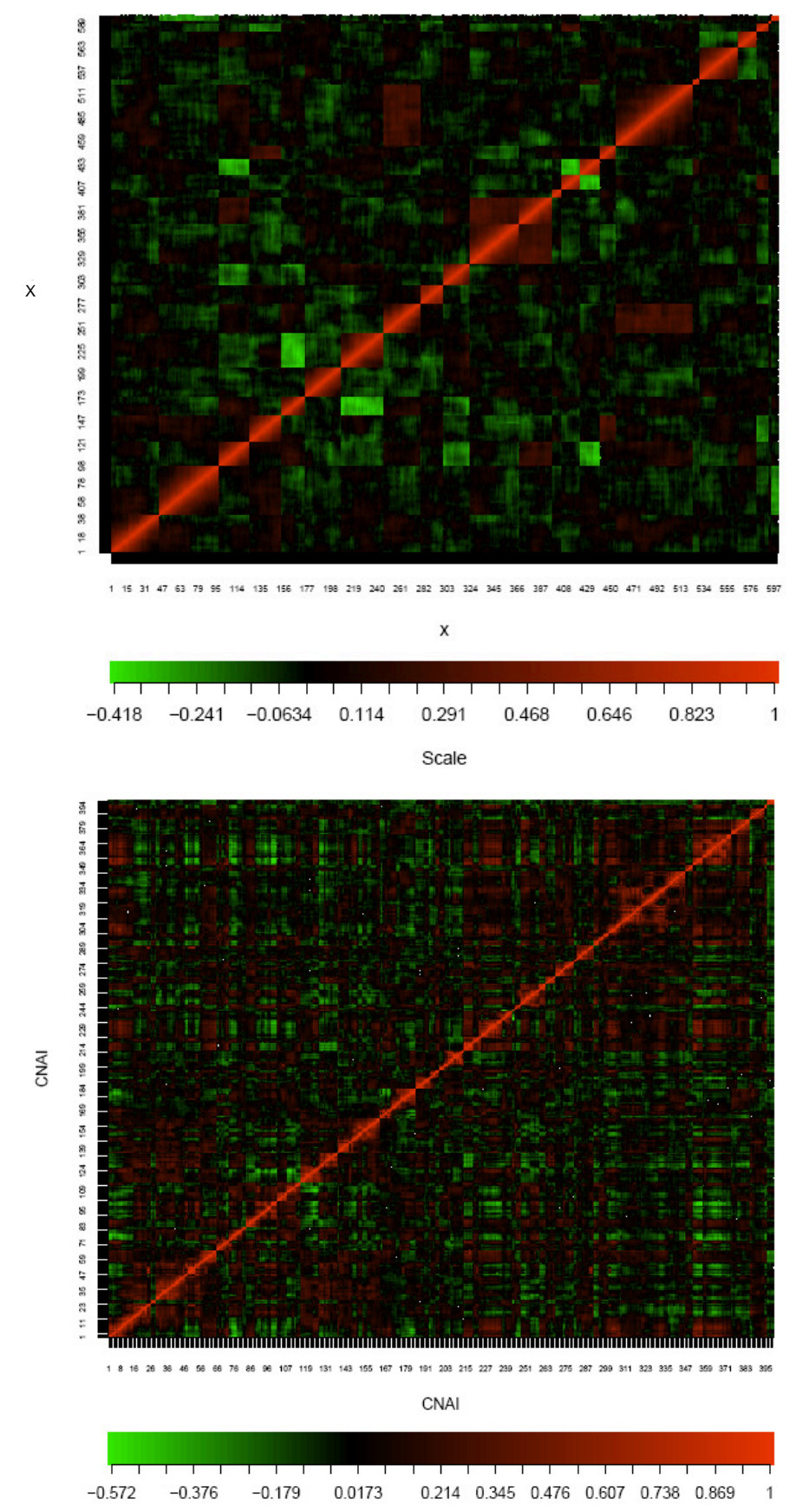

FIG S-2. Heatmaps of the sample correlations among predictors. Top panel: simulated data; Bottom panel: real data 


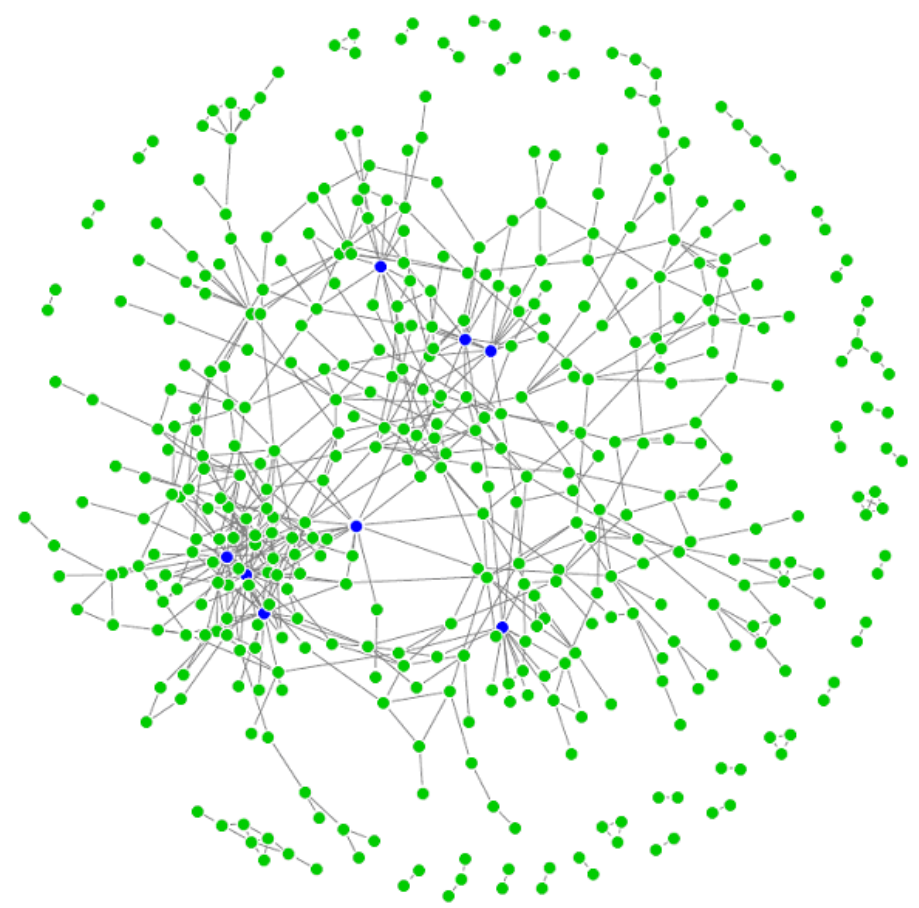

(a) Exp.Net.664: Inferred network for the 654 breast cancer related genes (based on their expression levels) by space. Nodes with degrees greater than ten are drawn in blue.

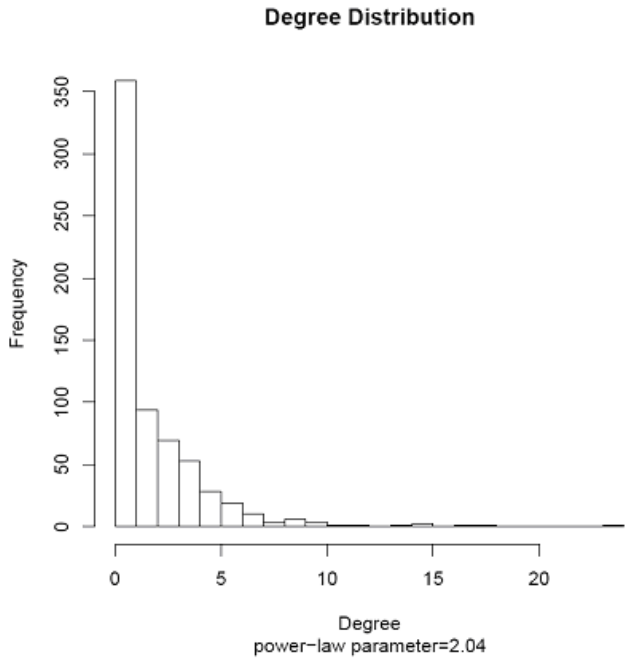

(b) Degree distribution of network Exp.Net.664.

FIG S-3. Inferred RNA interaction network. 


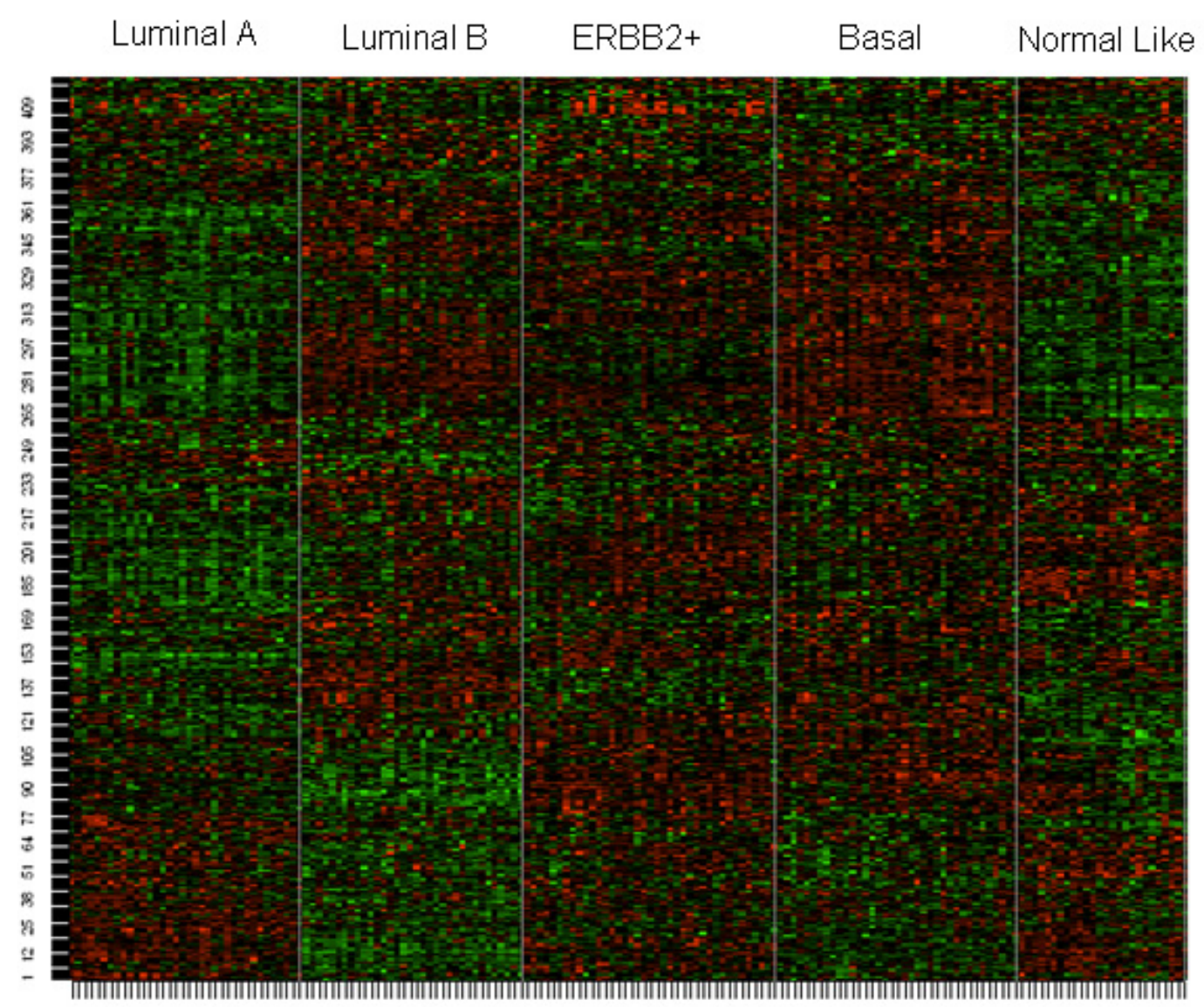

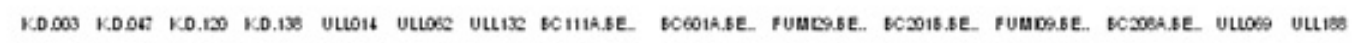
Sample

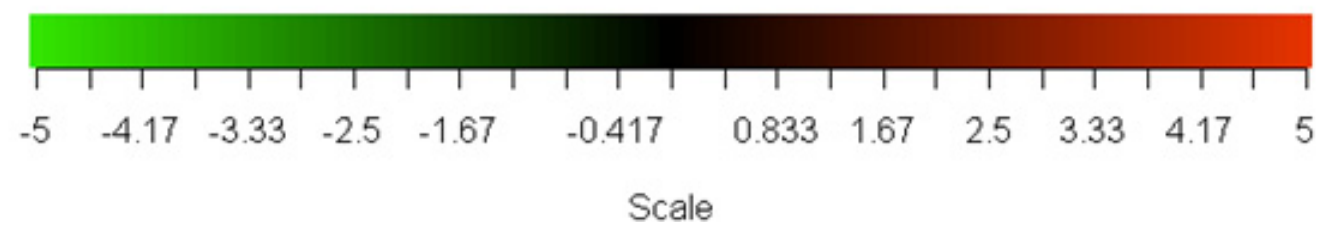

FIG S-4. Heatmap showing the expressions of the 449 intrinsic genes in the 172 breast cancer tumor samples. Each column represents one sample and each row represents one gene. The 172 samples are divided into 5 clusters (subtypes). From the left to the right, the 5 subtypes are: Luminal Subtype A, Luminal Subtype B, ERBB2-overexpressing subtype, Basal Subtype and Normal Breast-like Subtype. 